\title{
INVESTIGACIÓN/RESEARCH
}

\section{LA HORA DE LOS SECRETOS DE ELEONORA GABALDÓN: UNA APROXIMACIÓN AL DISCURSO DE GÉNERO}

\author{
María Elena Del Valle-de Villalba: Universidad Metropolitana de \\ Caracas. Venezuela. \\ manedelvalle@gmail.com
}

Nancy Elena Mejías-Briceño: Universidad Pedagógica Experimental Libertador en Caracas. Venezuela.

itadelvalle@hotmail.com

\section{RESUMEN}

Este trabajo presenta un análisis del discurso sobre los estereotipos reales e ideales que acerca del género construye Eleonora Gabaldón en su novela La Hora de los Secretos. La investigación se inserta dentro de un estudio mayor sobre el análisis de las producciones discursivas de las mujeres en latino-América. A partir de la realización del análisis de contenido de la novela y grupos de discusión, se analizará la presencia de estereotipos de género en el discurso de la autora con el objetivo de determinar hasta qué punto dichos estereotipos facilitan u obstaculizan la disposición de la mujer como elemento social desde la diferencia y se caracterizarán los roles, papeles y limitaciones asignadas a la misma en un determinado contexto histórico. Esto nos permitirá acercarnos a un concepto de género como frontera interna, es decir, como espacio de negociación y/o conflicto para las relaciones entre géneros; las que pueden ser entendidas como relaciones interculturales. Los resultados que se presentan son tentativos y se basan en la aplicación de las técnicas de análisis de contenido y de la realización de grupos de discusión. No se pretende su generalización, sino más bien abrir brechas de reflexión y análisis posteriores.

PALABRAS CLAVE: Discurso de Género - Funciones - Estereotipos Eleonora Gabaldón 


\title{
ELEONORA GABALDON'S LA HORA DE LOS SECRETOS: AN APPROACH TO THE GENDER DISCOURSE
}

\begin{abstract}
This paper presents an analysis of the discourse on the real and ideal stereotypes about gender constructs Eleonora Gabaldon's novel El tiempo de los secretos (The Time of Secrets). The research is embedded within a larger study on the analysis of the discursive production of women in Latin America. Since the completion of content analysis of the novel and discussion groups will analyze the presence of gender stereotypes in the discourse of the author in order to determine to what extent these stereotypes help or hinder the provision of women as social element from the difference and characterize the roles, roles and limitations assigned to it in a particular historical context. This will help us to a concept of gender as internal boundary, as a space for negotiation and / or conflict relations between genders, which can be understood as intercultural relations. The results are tentative and are based on the application of content analysis techniques and conducting focus groups. It is not intended to generalize, but rather to open reflexive and analysis.
\end{abstract}

KEY WORDS: Gender discurse - Roles - Stereotypes - Eleonora Gabaldón

\section{INTRODUCCIÓN}

Los estudios que actualmente se realizan sobre las relaciones interculturales han soslayado en su mayoría el conflicto entre los géneros como tópico de investigación, aunque existen de hecho líneas de investigación en las universidades sudamericanas que dan cuenta de este importante tópico, tanto desde el punto de vista de los estudios de género como desde el punto de vista del discurso como construcción social (Berelson, 1952). A nosotras, en este caso en particular nos interesa el estudio relacional de género entre hombres y mujeres, la perspectiva comparativa y dialógica entre ellos, y sobre todo la dimensión de articulación, de puente que, desde el discurso, pueda ser objetivada en los discursos literarios de mujeres en latino-América. Esto implica que, no abordaremos el tema de género desde la tradicional postura de la denuncia, sino que intentaremos estudiar las formas que adquiere el discurso de la Mujer, en este caso de la autora sobre las relaciones de género, con el fin de ubicar y describir las competencias interculturales de género que permiten o no la interacción entre hombres 
y mujeres, a partir de la descripción de los estereotipos presentes en el discurso de estos sujetos.

\section{METODOLOGÍA}

El conflicto entre géneros y la manera como este conflicto se dibuja a través de diferentes producciones discursivas es un problema social emergente que actualmente es abordado desde diversas aristas para poder comprender sus posibles implicaciones en la infinidad de ámbitos en los que se da. Por ello resulta consideramos necesario consolidar vetas de investigación empírica que sin desdeñar los esfuerzos y logros que en el campo de lo teórico han rendido frutos a lo largo de ya casi medio siglo, nutran el vasto suelo de esta discusión tan necesaria. De esta manera, esta investigación se emprende desde la complejidad y soporte teórico de un grupo de investigación que realiza aproximaciones al discurso de género en la literatura latino-Americana.

Lo anterior da cuenta de una perspectiva conceptual que contempla la dimensión objetiva y subjetiva de la realidad social dibujada en las novelas latinoamericanas: la primera se puede concebir a partir de los clivajes o condiciones estructurales, tangibles de los sujetos (clase, género, edad, religión y toda la gama de capitales propuesta por Bourdieu ${ }^{1}$ ) descritas por la autora en la novela; la segunda se aborda a partir del concepto de habitus, hábito y representaciones simbólicas, que nos permiten aproximarnos a lo intangible intercultural, y al conjunto de disposiciones en torno a las cuales se puede o no articular una definición a los roles y papeles asignados a la mujer en un momento histórico.

Los ámbitos considerados para el análisis son las relaciones interpersonales de la protagonista de la novela, en las que entran tanto relaciones familiares y relaciones afectivas de tipo amical y/o amoroso; las relaciones al interior y desde el interior de los espacios sociales, y por último, el ámbito de lo social donde tienen su origen $\mathrm{y} / \mathrm{o}$ consolidación el conjunto de creencias que sustentan los estereotipos de género. La vida de la mujer en Venezuela en la década de los años 60 es caracterizada por la autora describiendo los papeles, roles, limitaciones, permisos y prebendas derivados de cada uno de ellos.

La novela seleccionada para el estudio es: La Hora de los Secretos de Eleonora Gabaldón. Dicha novela pertenece a la Colección de la Biblioteca Andina y es editada por Planeta en el año de 1996. La autora:

\footnotetext{
${ }^{1}$ Bourdieu, Pierre (1988a) Cosas dichas. Buenos Aires: Gedisa.

Bourdieu, Pierre (1988b) La distinción. Madrid: Taurus.

Bourdieu, Pierre (1999) El espacio para los puntos de vista, en Revista Proposiciones, núm. 29: Historias y relatos de vida. Investigación y práctica en las ciencias sociales. Santiago de Chile: Ediciones Sur, p. 12-14.
} 
Eleonora Gabaldón, Socióloga, Investigadora en el campo de las ciencias sociales, narradora, siendo algunas de sus publicaciones: La /deología Política de Cipriano Castro y el Problema del Poder. (Publicaciones del Congreso de la República). El Pensamiento Político de Guzmán Blanco (Congreso de la República), Las Elecciones de 1835, La elección de José María Varga (FUNRES); La Constitución de 1858, (FUNRES, ACADEMIA DE LA HISTORIA), La Constitución de 1830 (FUNRES), El Discurso de la Unidad, 1900 (Fundación CELARG) y La Hora de los Secretos (Novela, Editorial Planeta), producción esta última, objeto de nuestro análisis.

En esta historia las acciones se producen en diferentes épocas que van desde el Gobierno de Gómez (1908-1935) hasta insipiente democracia, describiendo en cada momento histórico las variaciones en los supuestos culturales relacionados con la mujer. La narración se produce en planos que se mueven en los recuerdos de Aparecida, personaje central de esta historia. Así como las voces que le hacen presente a sus ancestros, recordándole sus máximas morales sobre el comportamiento esperado por la sociedad, de un hombre y una mujer.

\section{ANÁLISIS Y DISCUSIÓN}

La familia de Aparecida es de origen andino, se vienen a Caracas buscando un mejor lugar para su familia y van adaptándose poco a poco a las costumbres de Caracas. Aparición va a contar en forma retrospectiva su historia y para ello reúne a sus ancestros -ya muertospara contarles las dificultadas por las que ha pasado su personalidad enclavada en el ayer y habitante de un tiempo de grandes transformaciones, donde la mujer es protagonista de grandes cambios.

Aparición nace en los años 40 , tiempos de guerra. De buena familia, sin fortuna pero de mucha educación y dignidad educaron a la niña en un colegio de monjas, la formaron en el temor a Dios, y las costumbres más austeras y formales. De escasa autoestima, sumamente tímida la protagonista se refugia en su imaginación apartándose de sus compañeras. Vive con veneración el matrimonio de sus padres. La prisión del padre, (por su posición frente al gomecismo) las dificultades económicas que son solventadas por la entereza de la madre caracterizan el contexto narrativo que la prepara cada día para cumplir su papel en la sociedad. Como niña decenté y apegada a las buenas costumbres, recatada y modesta se adecua a lo que su madre le va señalando amorosa pero firmemente. Idealiza la figura masculina en el tío que le hablaba de sus atributos que aparentemente nadie notaba. Aparición admiraba a su madre y quería ser amada como ella lo era. Sin infidelidades por parte de su padre los tíos en la sobremesa hablaban de lo que debía ser una muchacha: preparar buen pan y dedicada a su hogar. Los hombres podían trasgredir las normas - porque eran hombreslas mujeres deberán perdonarles las infidelidades por la misma razón. 
Aparición va a las fiestas y su madre le recuerda que por ahora los hombres que conocerá podrán ser "sólo a-mi-gos". Ella lo acepta como todo lo que su madre dice. Cuando tiene novio (Santiago) lleva un noviazgo casto, pero no ausente de tentaciones carnales las que tiene que confesar. El matrimonio se realiza de acuerdo con las normas y costumbres y Aparición se siente bien como dueña de casa. Después de un tiempo Santiago cambia sus tranquilas costumbres por su incursión en la política. Le es infiel con una amiga pero ella lo perdona porque debe hacerlo. -son cosas de hombres- Aparecida se debate en una angustia existencial entre lo que siente como mujer y lo que vive como esposa. No entiende el afán arribista del marido pero se lo calla pasivamente. Santiago olvida sus ideales sociales que tanto respetaba y Aparición se convierte en un participante pasivo de la política y sus corruptelas. El llena la casa de objetos nuevos no necesarios, ella se involucra en esa feria de vanidades y derroches. Viajes, compra de apartamentos en el exterior hoy en Margarita vehículos nuevos, una camioneta para ella y sus hijos. Ya tienen cuatro.

Ella trata de olvidar sus sueños de esposa y compañera de su marido como había sido su madre, pero no se le da el milagro de sus padres. La madre aconseja aguantar, enamorarlo, complacerlo, todo para que no se busque otra. Es necesario mantener la apariencia de un hogar perfecto. Va al psicólogo que la escucha y le hace sentir que es una mujer de valía que debe quererse así misma. En él encuentra un apoyo cariñoso pero el médico eventualmente muere.

Otra vez entregada en su depresión busca una serie de actividades pero no la llenan. Le parece vacía la vida no encuentra salida, los hijos no la comprenden. Le da por la bebida. Consigue un empleo en una revista como cronista, y empieza a tener éxito Los hijos no la apoyan sin embargo continua. Viaja al extranjero con las delegaciones del gobierno y en una de sus salidas consigue a un matrimonio amigo. Ignacio y Maribel cuyo matrimonio es de conveniencia.

Ella se impacta con el marido y en otra oportunidad se encuentran y comienzan un romance de locura de encuentros fortuitos y escondidos. Ella abandona a sus hijos y se escapa muchas veces para encontrarse con el amante. Continúa su conflicto y se hacen presentes sus ancestros para recordarle sus deberes, pero no hace caso. Ha puesto en este amor de locura todo su interés. Se olvida de su trabajo y se dedica únicamente a vivir los contados momentos que él puede dedicarle. Todo lo entrega esta mujer por una migaja de tiempo y de amor. Llega un momento en que él es transadlo a un país lejano como embajador ella quiere seguirlo. Le pide tiempo. Su mujer se ha enterado de todo. Él prefiere cortar con ella. 
Otra vez se refugia en el alcohol y en sus diálogos con los desaparecidos, que le recuerdan -sobre todo las mujeres- que es así la vida de las "barraganas". Sin esperanzas, acompañada de su dolor y su fracaso conversa con su madre que en el último momento de su vida le dice que en algún lugar estará el amor que tanto espera. Es una historia trágica de una búsqueda del amor en una sociedad aparencial que ha ido dejando espacios a la mujer en los que tampoco ha logrado ser feliz. Atrapada en otras exigencias para poder ser libre y autosuficiente teniendo que pagar un alto precio.

\subsection{Categorías Emergentes}

Luego de realizar la lectura de la Novela y de aplicar las técnicas del análisis de contenido, emergieron en nuestro análisis las siguientes categorías que definen el comportamiento de la mujer como género.

\subsection{Comportamiento Societales descritos}

Según Fitcher ${ }^{2}$, la sociedad enjuicia cada acción de acuerdo con tres niveles de comportamiento, aplica las sensaciones y presiones correspondientes para corregirlos en caso de que estos niveles de comportamiento no sean satisfactorios:

a) Un comportamiento exigido, sin el cual no se puede desempeñar la función social; por ejemplo, se requiere que los estudiantes asistan a clases, realicen tareas y se sometan a exámenes, entre otras cosas.

b) Un comportamiento permitido, sobre el que la sociedad no establece reglas fijas y rígidas; por ejemplo, se permite fumar a las estudiantes fuera del salón de clase, que se organicen para formar círculos de estudio, etc.

c) Un comportamiento prohibido, contra el que la sociedad actúa imponiendo penas o sanciones; Por ejemplo, no se permite destruir el mobiliario del salón de clases, ni las instalaciones sanitarias de la escuela, etc.

El grado con que cada individuo se acomoda a las exigencias del primer nivel de comportamiento y evita el último, indica el grado en que desempeña su función social propia; también señala la medida en que la sociedad aprueba el desempeño de su función social y que tanto se le imponen sanciones de aprobación o desaprobación ( $p$. 56)

De esta manera los comportamientos societales ${ }^{3}$ descritos en la novela se categorizaron de la siguiente manera:

\footnotetext{
${ }^{2}$ Fichter, J oseph (1979). Sociología. Barcelona: Herder.

${ }^{3}$ Gómez Jara, Francisco A. (1989). Sociología. México: Porrúa.
} 
Tabla 1. Comportamientos societales descritos en la novela

\begin{tabular}{|c|c|c|c|}
\hline & $\begin{array}{c}\text { Comportamiento } \\
\text { Exigido: }\end{array}$ & $\begin{array}{l}\text { Comportamiento } \\
\text { Permitido: }\end{array}$ & $\begin{array}{l}\text { Comportamiento } \\
\text { Prohibido: }\end{array}$ \\
\hline & 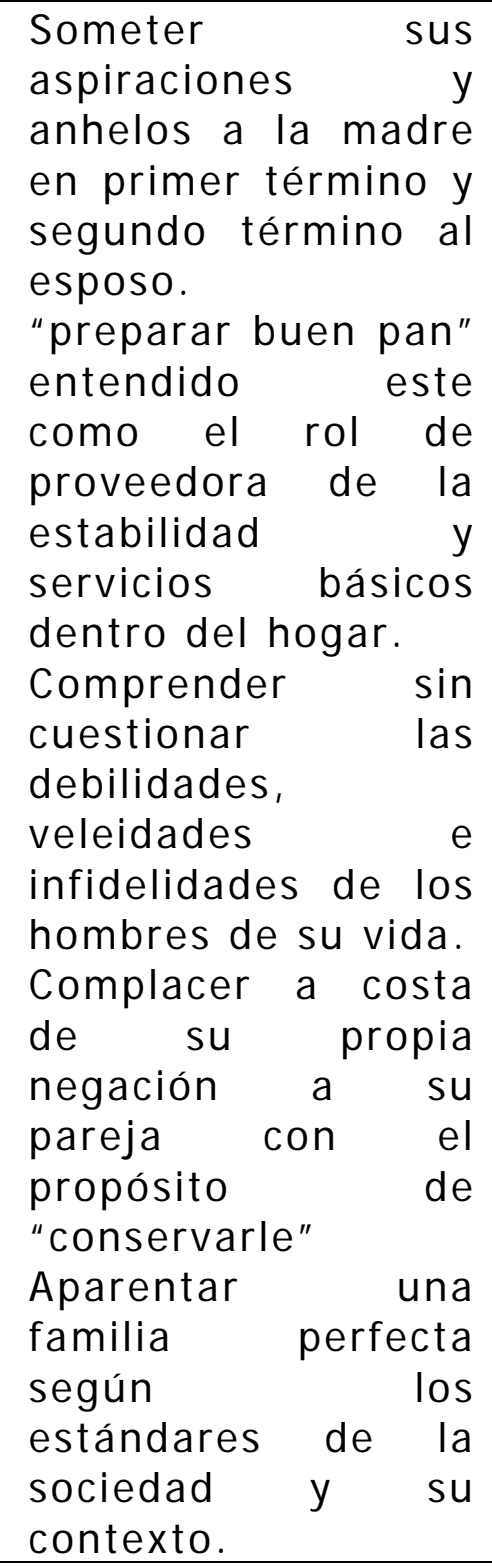 & $\begin{array}{l}\text { - Imagina un mundo } \\
\text { en el que es libre y } \\
\text { da rienda suelta a } \\
\text { la Aparición sin } \\
\text { ataduras. } \\
\text { - Sentir } \\
\text { inconformidad en la } \\
\text { intimidad de sus } \\
\text { espacios } \\
\text { experimentar el } \\
\text { vacío de sentir que } \\
\text { no complace a } \\
\text { nadie ni a ella } \\
\text { misma. } \\
\text { - La venganza a } \\
\text { través de la } \\
\text { infidelidad, la que } \\
\text { se permite como } \\
\text { otro espacio en la } \\
\text { que Aparición se } \\
\text { dibuja. }\end{array}$ & $\begin{array}{ll}\text { - } & \text { Cuestionar las } \\
& \text { figuras de } \\
\text { autoridad y sus } & \text { dineamientos } \\
\text { - Exigir espacios } \\
\text { para la auto- } \\
\text { realización } \\
\text { - Hacer realidad lo } \\
\text { que imagina. (al } \\
\text { menos notodo) }\end{array}$ \\
\hline
\end{tabular}

Fuente: Elaboración propia

\section{CONCLUSIONES}

Existen en la construcción discursiva de Eleonora Gabaldón elementos característicos e interesantes como: 
a) La denuncia implícita de las armaduras arquetipales (Jung, 1954) 45 a la que la sociedad de su tiempo sometía a la mujer como madre, esposa, hija, amante.

b) El dibujo de los personajes es solo emocional. Los rasgos físicos no se describen en lo absoluto.

c) El diálogo con las conciencias se imbrica como una malla moral a lo largo de la narración en la que sus voces le recuerdan "el deber ser" de conducta.

Esta novela constituye una radiografía crítica y descarnada de los limitantes sociales a los que la mujer venezolana se ve sometida voluntariamente o como producto de la coerción social. Las categorías generadas en este estudio se proponen como los puntos en los cuales puede fijarse la atención al momento de analizar un discurso narrativo.

\section{REFERENCIAS}

Berelson, B. (1952). Content analysis in communication research. Nueva York: The Free Press.

Bourdieu, P. (1988a) Cosas dichas. Buenos Aires: Gedisa.

Bourdieu, P. (1988b) La distinción. Madrid: Taurus.

Bourdieu, P. (1999). El espacio para los puntos de vista, en Revista Proposiciones, núm. 29. Historias y relatos de vida. Investigación y práctica en las ciencias sociales. Santiago de Chile: Ediciones Sur, p. 1214.

Fitcher, J. (1979) Sociología. Barcelona: Herder.

Gómez J. \& Francisco A. (1989). Sociología. México: Porrúa.

Jung, C. G. (1954a). Sobre el arquetipo con especial consideración del concepto del ánima. En C. G. Jung. (2002). Los arquetipos y 10 inconsciente colectivo. OC. 9. Barcelona: Trotta.

\footnotetext{
4J ung, Carl. G. (1954a). Sobre el arquetipo con especial consideración del concepto del ánima. En C. G. Jung. (2002). Los arquetipos y lo inconsciente colectivo. OC. 9. Barcelona: Trotta.

Jung, Carl. G. (1954b). Los aspectos psicológicos del arquetipo de la madre. En C. G. Jung. (2002). Los arquetipos y lo inconsciente colectivo. OC. 9. Barcelona: Trotta.

${ }^{5}$ Para Jung $(1954 a, 1954 b)$ la mujer ha sido representada por dos tipos de arquetipos: el arquetipo de la madre y del ánima. Éstos fueron definidos como ideas o formas preconcebidas que actuaban sobre los individuos determinando sus acciones y comportamientos. En este sentido, el arquetipo (J ung, 1941, p. 150): “... no sólo presenta algo que ha sido y que ha pasado hace tiempo sino también algo actual, es decir, no es sólo un residuo sino un sistema que sigue funcionando hoy y que está destinado a compensar o a corregir adecuadamente los inevitables unilateralismos y extravagancias de la conciencia".
} 
Jung, C.I G. (1954b). Los aspectos psicológicos del arquetipo de la madre, en C. G. Jung (2002) Los arquetipos y lo inconsciente colectivo. Barcelona: Trotta.

\section{María Elena Del Valle de Villalba}

Graduada de Profesora en la Universidad Pedagógica Libertador en el año 1992, con especialización en Historia Económica y Social de Venezuela en la Universidad Santa María, con Maestría en Historia Económica y Social de Venezuela. Autora de tesis con mención publicación y suma cum laude. Doctora en Ciencias de la Educación en el Pedagógico de Caracas (Venezuela).

\section{Nancy Elena Mejías}

Graduada como especialista en Literatura, con especialización en Gerencia en la Universidad Pedagógica Experimental Libertador de Caracas (Venezuela). 